\title{
Ensuring Structural Constraints in Graph-Based Models with Type Inheritance
}

\author{
Gabriele Taentzer ${ }^{1}$ and Arend Rensink ${ }^{2}$ \\ 1 Computer Science Department, Technical University of Berlin, \\ Franklinstr. 28/29, 10587 Berlin, Germany \\ 2 Computer Science Department, University of Twente, \\ P.O. Box 217, 7500 AE Enschede, The Netherlands
}

\begin{abstract}
Graphs are a common means to represent structures in models and meta-models of software systems. In this context, the description of model domains by classifying the domain entities and their relations using class diagrams or type graphs has emerged as a very valuable principle. The constraints that can be imposed by pure typing are, however, relatively weak; it is therefore common practice to enrich type information with structural properties (such as local invariants or multiplicity conditions) or inheritance.

In this paper, we show how to formulate structural properties using graph constraints in type graphs with inheritance, and we show how to translate constrained type graphs with inheritance to equivalent constrained simple type graphs. From existing theory it then follows that graph constraints can be translated into preconditions for productions of a typed graph transformation system which ensures those graph constraints. This result can be regarded as a further important step of integrating graph transformation with object-orientation concepts.
\end{abstract}

\section{Introduction}

Graphs and graphical representations play a central role in modeling and meta-modeling of software systems. Graphs are used to describe essential structures of entities and their relations. Their representation ranges from simply formatted, graph-like notations such as class diagrams, Petri nets, automata, etc. to more elaborated diagram kinds such as message sequence charts and to more application-specific notations for modeling, e.g., for industrial production processes.

In graph-based modeling and meta-modeling, graphs are used to define the static structure, such as class and object structures, data base schemes, as well as visual symbols and interrelations, i.e., visual alphabets and sentences. Graph manipulations describe the dynamic changes of these structures. Classifying the possible entities and interrelations in static system structures or visual language constructs isas a valuable principle for the description of model domains. In the object-oriented approach, class diagrams are the basic means to specify classification structures; e.g., in UML (Unified Modeling Language) [11] for software systems and MOF (Meta Object Facility) [11] for visual language specification. When applying graph transformation for modeling or metamodeling, type graphs are used to classify graph nodes and edges. 
One of the main principles to handle complex classification structures comes from the object-orientation paradigm: class inheritance enhances the typing principle by adding more abstract types on top of the ones concretely used in the (meta)models. Inheritance allows much more compact representations by reducing redundancy. The principle of inheritance has been carried over and formalized for graph transformation in [2]; there we have shown that node inheritance in typed graph transformation leads to a denser form of a graph transformation system, by a simular reduction of redundancy.

The power of pure typing to describe and constrain the static structure is, however, relatively weak (and is not enhanced by inheritance). It is therefore common practice to enrich type information with structural properties which further constrain the correct instances. A typical class of such structural properties are multiplicity conditions, which restrict correctly typed structures to those where the numbers of entities and interrelations are within given ranges. Further constraints can be local invariants which require, e.g., the existence or non-existence of certain substructures. In class diagrams, some of these constraint kinds are built-in, like multiplicities, while others have to be stated by separate constraints using, e.g., OCL [11]. On the other hand, typed graphs can be equipped with graph constraints, as proposed first in [9], which can be used to describe a variety of local invariants. Note, however, that graph constraints have so far been studied for flat graphs only (i.e., without node type inheritance).

The object-oriented and graph transformation approaches can be integrated by identifying classes with node types, and associations with edge types. In this way, class inheritance naturally corresponds to node type inheritance. In this paper we show how to express multiplicities and edge inheritance by graph constraints over type graphs with inheritance. Furthermore, we give a translation of constrained type graphs with inheritance to constrained flat type graphs. From existing theory [6] it then follows that graph constraints can be translated into (necessary and sufficient) pre-conditions for typed graph transformation rules. Our result can be regarded as a necessary further step of integrating graph transformation with object-orientation concepts. Application areas for the resulting theory are for instance: operational semantics for object-oriented systems as in [4] (leading to a theory of behavioral verification) and refactoring as in [10] (leading to a formal underpinning). We use a running example from the former area.

The paper is organized as follows: In the next section, we recall type graphs with node type inheritance as introduced in [2]; this will be the basis for further development. In Section 3 defined graph constraints over type graphs with inheritance and presents a translation to constraints over simple type graphs. Then Section 4 shows that multiplicities and edge inheritance are expressible by graph constraints. Section 5 describes how graph constraints can be ensured by typed graph transformation systems, reusing and extending the results in [6]. All proofs are omitted due to lack of space.

\section{Type Graphs with Node Type Inheritance}

The basic idea for specifying node type hierarchies is to introduce a special kind of (directed) edges, called inheritance edges, into type graphs. The source node of an inheritance edge is said to be a sub-type of the target node, which is called the former one's super-type. Moreover, nodes are marked either as concrete or abstract; we will 


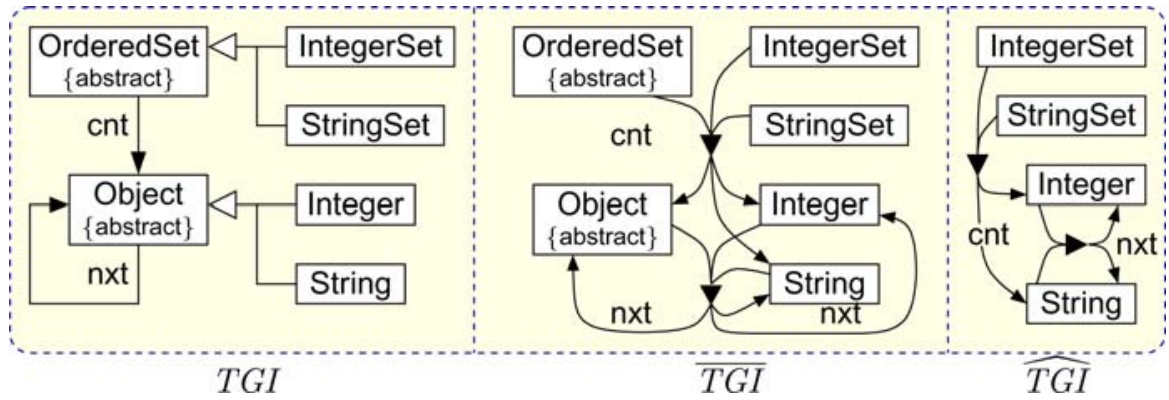

Fig. 1. A sample type graph with node type inheritance, and its abstract and concrete closure

see that only concrete type nodes can have direct instances. In host graphs only nodes of concrete types shall occur, while graphs in rules may contain nodes of both types.

Definition 1 (type graph with inheritance). A type graph with inheritance is a triple $T G I=(T G, I, A)$ consisting of a type graph $T G=(N, E, s, t)$ (with a set $N$ of nodes, a set $E$ of edges, source and target functions $s, t: E \rightarrow N)$, an acyclic inheritance relation $I \subseteq N \times N$, and a set $A \subseteq N$, called abstract nodes. For each $x \in N$, the inheritance clan is defined by $\operatorname{clan}_{I}(x)=\left\{y \in N \mid(y, x) \in I^{*}\right\}$, where $I^{*}$ is the reflexive-transitive closure of $I$.

Example 1. As sample type graph we use TGI in Fig.11 This describes a special kind of sets, namely ordered sets, which contain a number of objecs (indicated by cnt-edges from OrderedSet-nodes to Object-nodes) which can be put into some order (indicated by nxt-edges among the object). We consider two possible specializations of ordered sets, namely StringSet and IntegerSet, which are intended to contain Strings and Integers, respectively. Note that the type graph by itself does not yet enforce this constraint: that is, it does not rule out that StringSets contain also Integers, and vice versa.

To benefit from the existing theory of graph transformation [5], which does not recognize the notion of inheritance, we define the flattening or closure of type graphs with inheritance to ordinary ones.

Definition 2 (Closure of type graph with inheritance). Let $T G I=(T G, I, A)$ be a type graph with inheritance, and let $T G=(N, E$, src, tar $)$. The abstract closure of TGI is the graph $\overline{T G I}=(N, \bar{E}, \overline{s r c}, \overline{\operatorname{tar}})$ with

- $\bar{E}=\left\{\left(n_{1}, e, n_{2}\right) \mid e \in E, n_{1} \in \operatorname{clan}_{I}(\operatorname{src}(e)), n_{2} \in \operatorname{clan}_{I}(\operatorname{tar}(e))\right\}$;

$-\overline{\operatorname{src}}\left(\left(n_{1}, e, n_{2}\right)\right)=n_{1}$;

- $\overline{\operatorname{tar}}\left(\left(n_{1}, e, n_{2}\right)\right)=n_{2}$.

The concrete closure of TGI is the graph $\widehat{T G I}=\left.\overline{T G I}\right|_{N-A}$

\footnotetext{
${ }^{3}$ Given a graph $G=(N, E, s, t)$ and a set $X \subseteq N$, we denote by $\left.G\right|_{X}$ the sub-graph $\left(X, E_{X}=\right.$ $\left.\{e \in E \mid s(e), t(e) \in X\},\left.s\right|_{E_{X}},\left.t\right|_{E_{X}}\right)$.
} 


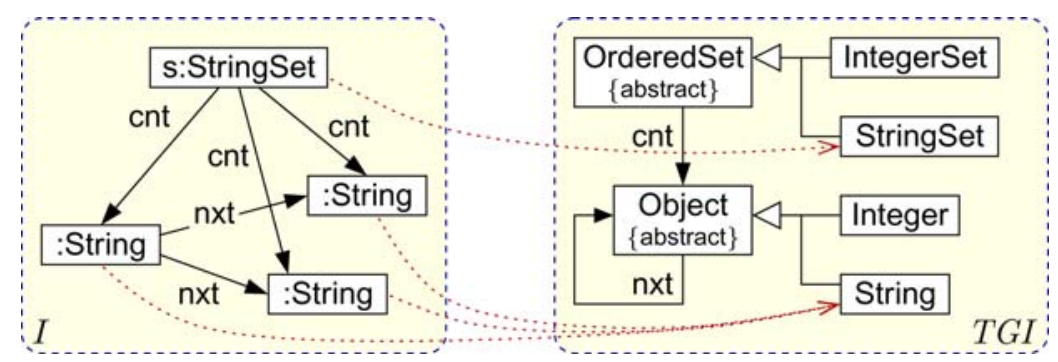

Fig. 2. Sample clan-typed graph

Example 2. Fig. 1 also shows the abstract and concrete closure of the type graph with inheritance $T G I$. Please note that for better readability of the closures, the edge types are bundled using auxiliary nodes. Note that the inheritance edges are no longer present in the closure, and the abstract node types and adjacent edge types are absent from the concrete closure. Instead, for all combinations of corresponding sub-types a new edge type is inserted - including those which do not follow our intuition, like edge type nxt between String and Integer. We will use structural graph properties in addition to rule out those unwanted structures.

The distinction between the abstract and the concrete closure of a type graph is necessary, since they give rise to different instances. We will define abstract graph transformation rules of which the left hand and right hand sides are typed over the abstract closure (see Sect. 5], whereas ordinary host graphs and concrete rules are typed over the concrete closure.

Definition 3 (instance graph). An abstract instance graph $\left(G, t p_{A}\right)$ of a type graph with inheritance TGI is an instance graph of $\overline{T G I}$; i.e., $t p_{A}: G \rightarrow \overline{T G I}$. Analogously, a concrete instance graph $\left(G, t p_{C}\right)$ of TGI is a graph typed over $\widehat{T G I}$.

Note that, due to the canonical inclusion $i n c_{T G}: \widehat{T G I} \hookrightarrow \overline{T G I}$, all concrete instance graphs are abstract instance graphs. The construction of the closure in Def. 2 gives rise to a characterization of instance graphs directly on type graphs with inheritance. Namely, instance graphs can be typed over the type graph with inheritance by a pair of functions, from nodes to node types and from edges to edge types, respectively. This pair of functions does not constitute a graph morphism, but will be called clan morphism; it uniquely characterizes the type morphism into the flattened type graph.

Definition 4 (clan morphism). Let $T G I=(T G, I, A)$ be a type graph with inheritance. A clan-morphism from $G$ to $T G I$ is a pair ctp $=\left(\operatorname{ctp}_{N}: N_{G} \rightarrow\right.$ $\left.N_{T G}, \operatorname{ctp}_{E}: E_{G} \rightarrow E_{T G}\right)$ such that for all $e \in E_{G}$ the following holds:

- $\operatorname{ctp}_{N} \circ s_{G}(e) \in \operatorname{clan}_{I}\left(s_{T G} \circ \operatorname{ctp}_{E}(e)\right)$ and

- $\operatorname{ctp}_{N} \circ t_{G}(e) \in \operatorname{clan}_{I}\left(t_{T G} \circ \operatorname{ctp}_{E}(e)\right)$.

$(G, c t p)$ is called a clan-typed graph. ctp is called concrete if $\operatorname{ctp}_{N}^{-1}(A)=\emptyset$.

Example 3. Fig. 2 shows a sample instance graph typed over TGI of Fig. 11 The edge typing is not shown explicitly, but follows uniquely from the node typing. The typing is 
done by a clan morphism which maps each node to its node type and each edge to an edge type between potentially more abstract node types holding the source and target types of the instance edge in their clans.

Proposition 1 (universal clan morphism, see [1]). Let $T G I=(T G, I, A)$ be a type graph with inheritance. There is a universal clan morphism $u_{T G}: \overline{T G I} \rightarrow T G$ such that for each clan morphism ctp : $G \rightarrow T G$ there is a unique graph morphism tp: $G \rightarrow \overline{T G I}$ with $u_{T G} \circ t p=c t p$.

We often write $G$ for the clan-typed graph $\left(G, c t p_{G}\right)$. To formalize the relationship between abstract and concrete rules (see Sect. 5), we introduce the notion of type refinement. This imposes an order over the set of clan morphisms of a given instance graph: one clan morphism is said to be finer than another if it assigns more concrete node types to the instance graph nodes.

Definition 5 (type refinement and typed graph morphism). Let $T G I=(T G, I, A)$ be a type graph with inheritance, and let ctp, ctp ${ }^{\prime}: G \rightarrow T G$ be clan typings. ctp is a refinement of $c t p^{\prime}$, denoted $c t p \leq c t p^{\prime}$, if

- $\operatorname{ctp}_{N}(n) \in \operatorname{clan}_{I}\left(\operatorname{ctp}_{N}^{\prime}(n)\right)$ for all $n \in N_{G}$, and

- $c t p_{E}=c t p_{E}^{\prime}$.

Given clan-typed graphs $\left(G, c t p_{G}\right)$ and $\left(H, c t p_{H}\right)$ over TGI, a morphism $g: G \rightarrow H$ is called type-refining if $g \circ c t p_{H} \leq c t p_{G}$, and type-preserving if $g \circ c t p_{H}=c t p_{G}$.

We write $\left(G, c t p_{G}\right) \leq\left(H, c t p_{H}\right)$ if $G=H$ and $c t p_{G} \leq c t p_{H}$. We write $g: G \rightarrow_{c} H$ to denote that $G$ and $H$ are both concrete and $g$ is an injective type-preserving morphism, and $g: G \rightarrow_{\mathrm{a}} H$ to denote that $g$ is an injective type-refining morphism. The following proposition states some facts regarding type-refining and type-preserving morphisms.

Proposition 2. Let $G, H$ be clan-typed graphs, and let $g: G \rightarrow H$ be type refining.

1. There is a unique clan-typed graph $K \leq G$ such that $g: K \rightarrow H$ is type-preserving;

2. For any clan-typed graph $K \geq G, g: K \rightarrow H$ is type-refining.

3. For any clan-typed graph $K \leq H, g: G \rightarrow K$ is type-refining.

\section{Structural Properties over Type Graphs with Inheritance}

The following definition extends the concept of graph constraints, originally introduced in [9] (where they are called consistency constraints). There are two points of change:

- We define constraints over concrete clan-typed graphs rather than ordinary typed graphs. However, this is not a real extension since (due to Prop.11, there is a one-toone correspondence between concrete clan morphisms and type morphisms to the concrete closure of the type graph.

- We allow constraints with multiple, disjunctively interpreted conclusions, rather than a single conclusion, as in [9,6]. This is a real extension, as it properly enlarges the set of properties expressible through graph constraints.

Whenever we mention "clan-typed graphs" in the following, we mean graphs with a clan morphism to some implicit, globally given type graph with inheritance $T G I$. 
Definition 6 (graph atoms and formulae). Let $L, G$ be clan-typed graphs, such that $G$ is concrete.

- A concrete [abstract] graph atom A over L is a tuple $\left(n: L \rightarrow_{\mathrm{c}} P, C o n\right)\left[\left(n: L \rightarrow_{\mathrm{a}}\right.\right.$ $P$, Con)], where $n$ is an injective type-preserving [type-refining] morphism, and Con is a set of injective type-preserving [type-refining] morphisms starting in P. If $L=\emptyset$ we also write $(P, C o n)$ for $A$.

- $A$ is said to be satisfied by an injective type-preserving [type-refining] morphism $m: L \rightarrow_{\mathrm{c}} G\left[m: L \rightarrow_{\mathrm{a}} G\right]$, denoted $m \models^{\mathrm{c}} A\left[m \models^{\mathrm{a}} A\right]$, if for all injective typepreserving [type-refining] morphisms $p: P \rightarrow_{\mathrm{c}} G\left[p: P \rightarrow{ }_{\mathrm{a}} G\right.$ ] such that $m=p \circ n$, there is $a(q: P \rightarrow C) \in C o n$ and an injective type-preserving [type-refining] morphism $c: C \rightarrow_{\mathrm{c}} G\left[c: C \rightarrow_{\mathrm{a}} G\right]$ such that $p=c \circ$ q. If $L=\emptyset$ (i.e., the empty graph) then we also write $G \models{ }^{\mathrm{c}} A\left[G \mid=^{\mathrm{a}} A\right]$.

- A concrete [abstract] graph formula $F$ over $L$ is a boolean formula over concrete [abstract] graph atoms over $L$. The satisfaction relation $\models^{\mathrm{c}}\left[\models^{\mathrm{a}}\right]$ is extended to graph formulae by defining the semantics of the boolean operators in the usual way. We call $F$ a constraint if $L=\emptyset$, and an application condition otherwise.

Example 4. Fig. 3 shows three atoms over the type graph with inheritance TGI in Fig.1 In this and later pictures we depict graph atoms $\left(L \rightarrow P,\left\{P \rightarrow C_{i}\right\}_{i}\right)$ more compactly as $L \rightarrow P \rightarrow\left\{C_{i}\right\}_{i}$.

- $A_{1}$ is satisfied by a morphism that selects an element without an outgoing nxt-edge;

- $A_{2}$ is satisfied by a graph if every OrderedSet is empty, i.e., contains no elements;

- $A_{3}$ is satisfied by a graph if for every OrderedSet and every pair of distinct elements contained in it, (at least) one element as an outgoing nxt-edge. Note that the graphs in the set on the right hand side are to be interpreted disjunctively.

$A_{2}$ and $A_{3}$ range over the same graph $L$, viz. the empty graph: in fact, they are constraints and can be combined into the formula $A_{2} \wedge A_{3}$. $A_{1}$, on the other hand, cannot be combined with $A_{2}$ or $A_{3}$ into one formula, since they are atoms over different graphs.

We can now define the flattening of an abstract atom and an abstract formula.

Definition 7 (flattening). Let $K, L$ be clan-typed graphs such that $K \leq L$ and $K$ is concrete.

- For any abstract graph atom $A=\left\langle n: L \rightarrow_{\mathrm{a}} Q\right.$, Con $\rangle$, the $K$-flattening of $A$ is defined by:

$$
\begin{aligned}
\operatorname{flat}_{K}(A) & =\bigwedge\left\{\left(n: K \rightarrow_{\mathrm{c}} P, \text { flat }_{P}(\text { Con })\right) \mid P \leq Q\right\} \\
\text { flat }_{P}(\text { Con }) & =\left\{q: P \rightarrow_{\mathrm{c}} C \mid\left(q: Q \rightarrow_{\mathrm{a}} D\right) \in \text { Con }, C \leq D\right\} .
\end{aligned}
$$

- For any abstract graph formula $F$ over $L$, the $K$-flattening flat ${ }_{K}(F)$ is defined by replacing each abstract graph atom $A$ occurring in $F$ by the corresponding $K$-flattening flat $_{K}(A)$.

In the next secion we give some examples of flattening. The following theorem is the main contribution of this paper. It states that satisfaction of an abstract atom or formula over an abstract clan-typed graph $L$ by a type-refining morphism $m: L \rightarrow_{\mathrm{a}} G$ is equivalent to satisfaction of the flattening of that atom or formula with respect to the concrete clantyped graph $K \leq L$ for which $m: K \rightarrow_{\mathrm{c}} G$ is type-preserving (which uniquely exists due to Prop.2.1). This allows us to re-use existing theory on concrete graph formulae. 


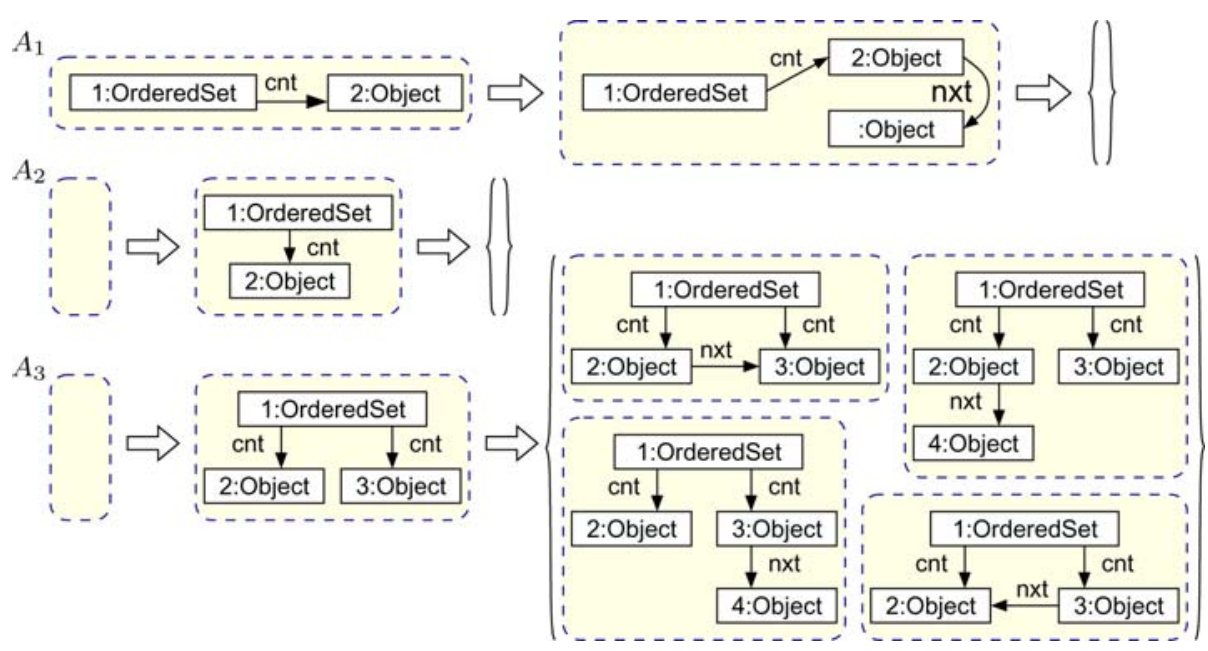

Fig. 3. Three example graph atoms

Theorem 1 (flattening of abstract graph formulae). Let $K, L, G$ be clan-typed graphs such that $K \leq L$, and let $m: K \rightarrow_{\mathrm{c}} G$. For any abstract graph atom A and graph formula $F$ over $L$ the following holds:

$$
\begin{aligned}
& \left(m: L \rightarrow_{\mathrm{a}} G\right) \models{ }^{\mathrm{a}} A \text { iff }\left(m: K \rightarrow_{\mathrm{c}} G\right) \models{ }^{\mathrm{c}} \operatorname{flat}_{K}(A) \\
& \left(m: L \rightarrow_{\mathrm{a}} G\right) \models{ }^{\mathrm{a}} F \text { iff }\left(m: K \rightarrow_{\mathrm{c}} G\right) \models{ }^{\mathrm{c}} \operatorname{flat}_{K}(F) .
\end{aligned}
$$

The proof relies on the fact that the flattening defined in Def.77 "predicts" all concrete ways in which the abstract atom and formula could be satisfied, by taking conjunctions resp. disjunction over all concrete instance graphs that are $\leq$-predecessors of the abstract premises and conclusions.

\section{Multiplicities and Edge Inheritance as Graph Formulae}

In this section we show that two existing classes of constraints on type graphs with inheritance can be translated to abstract graph formulae. This serves to give some intuition about graph formulae, and to demonstrate that they are expressive enough to cover practically useful examples. (It should be noted, however, that there are many graph constraints that do not fall into either of these classes: for instance, $A_{3}$ in Fig. 3 cannot be expressed through multiplicities or edge inheritance.

Multiplicities. By enriching a type graph with multiplicities we can restrict the class of instance graphs to those which are not only correctly typed but also satisfy additional constraints concerning the number of nodes and edges for each type. These constraints are expressed using so-called multiplicities.

Definition 8 (multiplicities). A multiplicity is a pair $[i, j] \in \mathcal{N} \times(\mathcal{N} \cup\{*\})$ with $i \leq j$ or $j=*$. The set of multiplicities is denoted Mult. The special value $*$ indicates that 
the maximum number of nodes or edges is not constrained. For an arbitrary finite set $X$ and $[i, j] \in$ Mult, we write $|X| \in[i, j]$ if $i \leq|X|$ and either $j=*$ or $|X| \leq j$.

As usual, we use multiplicities to decorate the nodes and edges of type graphs. For the nodes, the multiplicity indicates the total number of instances; for the edges, we use multiplicities expressing the number of incoming, respectively outgoing edges for each target, respectively source instance.

Definition 9 (Type graph with multiplicities). A type graph with multiplicities is a tuple $T G M=\left(T G I, m_{N}, m_{s r c}, m_{t a r}\right)$ consisting of a type graph with inheritance TGI and additional functions $m_{N}: N_{T G I} \rightarrow$ Mult, called node multiplicity function, and $m_{\text {src }}, m_{t a r}: E_{T G I} \rightarrow$ Mult, called edge multiplicity functions.

The satisfaction of multiplicity constraints is expressed by counting inverse images with respect to the clan typing.

Definition 10 (Semantics of type graphs with multiplicities). A clan-typed graph $G$ over $T G I=(T G, I, A)$ is said to satisfy a type graph with multiplicities ( TGI, $\left.m_{N}, m_{\text {src }}, m_{\text {tar }}\right)$ if the following conditions hold:

- for all $n \in N_{T G},\left|\operatorname{ctp}_{G}^{-1}\left(\operatorname{clan}_{I}(n)\right)\right| \in m_{N}(n)$;

- for all $e \in E_{T G}$ and $p \in \operatorname{ctp}_{G}^{-1}\left(\operatorname{clan}_{I}(\operatorname{src}(e))\right),\left|\operatorname{ctp}_{G}^{-1}(e) \cap \operatorname{src}_{G}^{-1}(p)\right| \in m_{\text {tar }}(e)$;

- for all $e \in E_{T G}$ and $p \in \operatorname{ctp}_{G}^{-1}\left(\operatorname{clan}_{I}(\operatorname{tar}(e))\right),\left|\operatorname{ctp}_{G}^{-1}(e) \cap \operatorname{tar}_{G}^{-1}(p)\right| \in m_{\text {src }}(e)$.

We now show how a type graph with multiplicities $T G M$ can be translated to an abstract graph formula that is satisfied by precisely those clan-typed graphs that also satisfy $T G M$. In order to do that, we introduce two special types of graphs: for all $i \in \mathcal{N}$,

- For all $n \in N, G_{i}^{n}$ is the graph consisting of $i$ distinct $n$-typed nodes.

- For all $e \in E, \mathcal{G}_{i}^{e, s r c}$ is the set of graphs with $i$ distinct $e$-typed edges and all source nodes glued together; dually, $\mathcal{G}_{i}^{e, t a r}$ is the set of graphs with $i$ distinct $e$-typed edges and all target nodes glued together.

Definition 11 (Multiplicities as abstract graph formulae). Given a type graph with multiplicities $T G M=\left(T G I, m_{N}, m_{\text {src }}, m_{\text {tar }}\right)$, we define

$$
F_{T G M}=\bigwedge_{n \in N_{T G I}} F_{n} \wedge \bigwedge_{e \in E_{T G I}}\left(F_{e}^{s r c} \wedge F_{e}^{\text {tar }}\right)
$$

where $F_{n}, F_{e}^{s r c}$ and $F_{e}^{\text {tar }}$ are abstract graph formulae defined as follows:

- $F_{n}$ regulates the node multiplicity of $n$. $\operatorname{Let}_{N}(n)=[i, j]$; then $F_{n}=A_{n \geq i} \wedge A_{n \leq j}$ if $j \neq *$ and $F_{n}=A_{n \geq i}$ otherwise, where

$$
\begin{aligned}
& A_{n \geq i}=\left(\emptyset,\left\{\emptyset \rightarrow G_{i}^{n}\right\}\right) \\
& A_{n \leq j}=\left(G_{j+1}^{n}, \emptyset\right) .
\end{aligned}
$$

- $F_{e}^{s r c}$ regulates the edge source multiplicity of e. Let $m_{\text {src }}(e)=[i, j]$; then $F_{e}^{s r c}=$ $A_{e \geq i}^{s r c} \wedge F_{e \leq j}^{s r c}$ if $j \neq *$ and $F_{e}^{s r c}=A_{e \geq i}^{s r c}$ otherwise, where

$$
\begin{aligned}
& A_{e \geq i}^{s r c}=\left(G_{1}^{\operatorname{tar}(e)},\left\{q^{\operatorname{tar}}: G_{1}^{\operatorname{tar}(e)} \rightarrow H \mid H \in \mathcal{G}_{i}^{e, t a r}\right\}\right) \\
& F_{e \leq j}^{s r c}=\bigwedge\left\{(H, \emptyset) \mid H \in \mathcal{G}_{j+1}^{e, t a r}\right\}
\end{aligned}
$$

with $q^{\text {tar }}$ mapping the sole node of $G_{1}^{\text {tar(e) }}$ to the unique target node of $H$. 


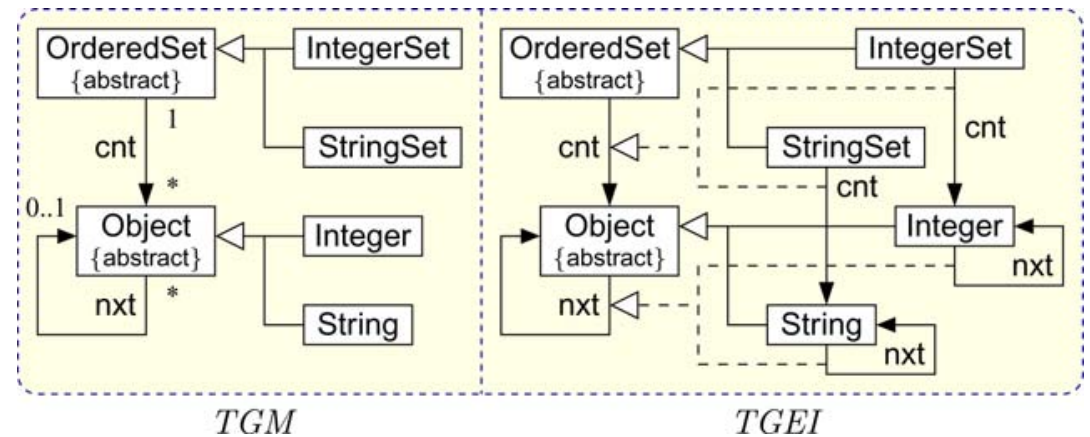

Fig. 4. Type graph with multiplicities, respectively edge inheritance

- $F_{e}^{\text {tar }}$ regulates the edge target multiplicity of e, and is the exact dual of $F_{e}^{\text {src }}$ (obtained by switching src and tar everywhere in the above definition).

The following theorem states that this formula indeed expresses the multiplicity semantics according to Def. 10. The proof is omitted here.

Theorem 2 (semantics of multiplicities). For all type graphs with multiplicity TGM and all graphs G clan-typed over TGI, G satisfies TGM (in the sense of Def. 10) if and only if $G \models{ }^{\mathrm{a}} F_{T G M}$.

Example 5 (multiplicity constraints). In Figure 4 (left hand side), the type graph TGI of Fig. 1 has been extended with multiplicities at edge types. For the notation of multiplicities we follow UML. Each object has always to belong to precisely one ordered set. This statement contains two constraints: a lower and an upper bound, which in this case are both equal to 1 . Vice versa, ordered sets are allowed to contain arbitrarily many objects, which is indicated by an asterisk. The nxt relation on objects is constrained to a partial order where at most one object is $n x t$, but each object may have arbitrarily many predecessors. This results in the five graph constraints depicted in Figure 5 (Note that we have omitted the empty initial graph.) The first constraint states that every object is contained in a set (which is a positive constraint), the next two that an object is not allowed to have two outgoing containment edges, neither to different nor to the same OrderedSet node (which are negative constraints), and the last two constraints (also negative) express that an object does not have two successor objects.

The next step is to flatten these graph constraints; i.e., we formulate graph constraints w.r.t. the concrete closure $\widehat{T G I}$ also given in Fig. 1] Some representatives of the flattened constraints are shown in Fig. 6. The first of these is the complete flattening of the first constraint in Fig. 5, the second and third show two of the four atomic constraints that constitute the flattening of the second constraint in Fig. 5 .

Edge Inheritance. As we have seen, node inheritance is used to formulate a compact type graph in the sense that edge types between super types stand for all combinations of edge types between their sub-types (including themselves). This might lead to a type graph with too loose type information concerning edges. In the following, we introduce edge type inheritance, which aims at restricting the combinations of sub-types allowed. 


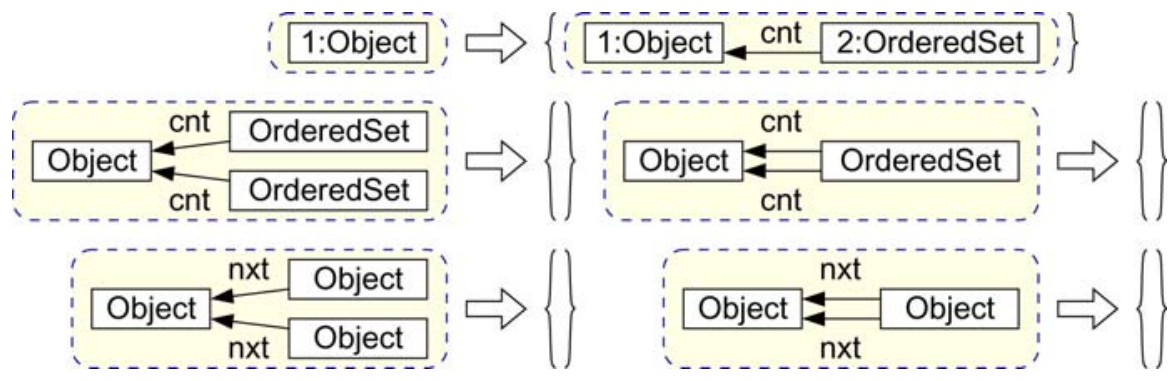

Fig. 5. Multiplicity constraints as abstract graph atoms

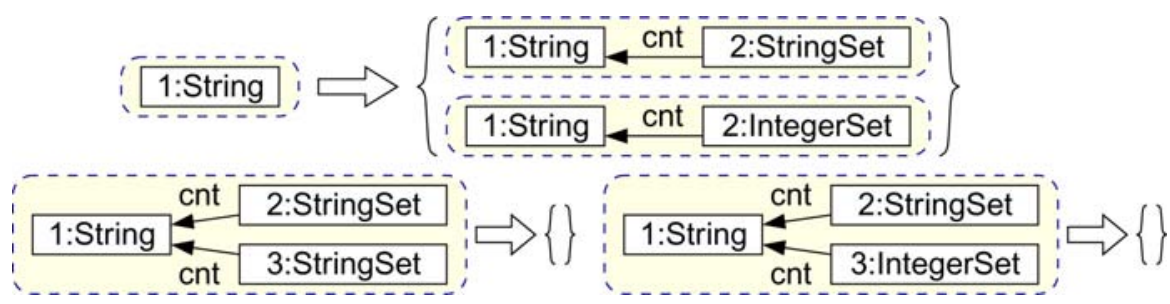

Fig. 6. Flattened multiplicity constraints

Definition 12 (type graph with edge inheritance). A type graph with edge inheritance is a tuple $(T G, I, A)$ where $I \subseteq(N \times N) \cup(E \times E)$ is an acyclic relation such that $T G I=\left(T G,\left.I\right|_{N}, A\right)$ is a type graph with (node) inheritance, and moreover, $(e, f) \in I \cap(E \times E)$ implies $\operatorname{src}(e) \in \operatorname{clan}_{I}(\operatorname{src}(f))$ and $\operatorname{tar}(e) \in \operatorname{clan}_{I}(\operatorname{tar}(f))$.

The idea is that if a type edge $e$ inherits from another type edge $f$, then $f$ can occur as an edge type only for concrete graph edges whose source and target node types are not in the clan of the source type, resp. target of $e$. The semantics of edge inheritance can either be expressed by redefining the closure, or directly as a constraint on the clan morphism. In other words, if the source or target node of an edge would allow $e$ as an edge type, then no proper super-type of $e$ may be used.

Definition 13 (semantics of type graphs with edge inheritance). A clan-typed graph $G$ over TGI is said to satisfy a type graph with edge inheritance (TG,I,A) for which $T G I=\left(T G,\left.I\right|_{N}, A\right)$ if for all $x \in E_{G}$ and $\left(e, \operatorname{ctp}_{G}(x)\right) \in I, \operatorname{ctp}_{G}\left(\operatorname{src}_{G}(x)\right) \notin$ $\operatorname{clan}_{I}\left(\operatorname{src}_{T G}(e)\right)$ and $\operatorname{ctp}_{G}\left(\operatorname{tar}_{G}(x)\right) \notin \operatorname{clan}_{I}\left(\operatorname{tar}_{T G}(e)\right)$.

We now construct an abstract graph formula which expresses the same constraint.

Definition 14 (edge inheritance as an abstract formula). Given a type graph with edge inheritance TGEI $=(T G, I, A)$, define $F_{T G E I}=\bigwedge_{(e, f) \in I_{E}} A_{e, f}^{\text {src }} \wedge A_{e, f}^{\text {tar }}$ where

$$
\begin{aligned}
& A_{e, f}^{s r c}=\left(G^{\operatorname{src}(e), f, \operatorname{tar}(f)},\left\{q_{e, f}: G^{\operatorname{src}(e), f, \operatorname{tar}(f)} \rightarrow G^{\operatorname{src}(e), e, \operatorname{tar}(e)}\right\}\right) \\
& A_{e, f}^{\operatorname{tar}}=\left(G^{\operatorname{src}(f), f, \operatorname{tar}(e)},\left\{q_{e, f}: G^{\operatorname{src}(f), f, \operatorname{tar}(e)} \rightarrow G^{\operatorname{src}(e), e, \operatorname{tar}(e)}\right\}\right) .
\end{aligned}
$$




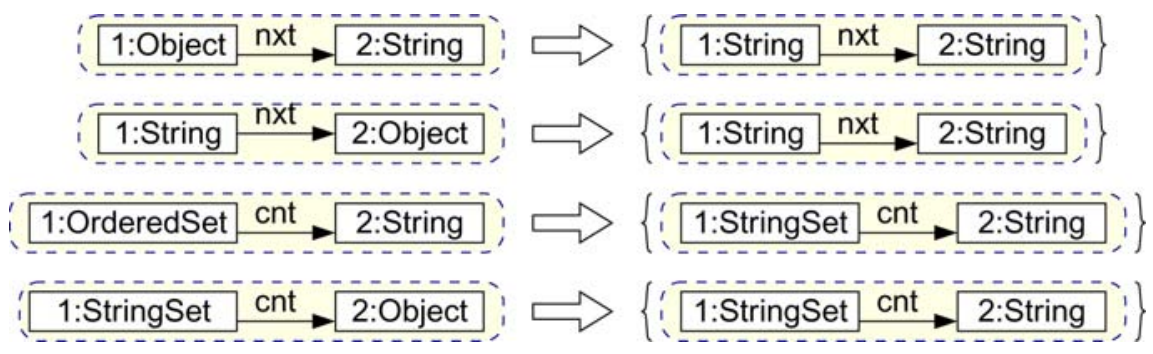

Fig. 7. Edge inheritance as graph constraints

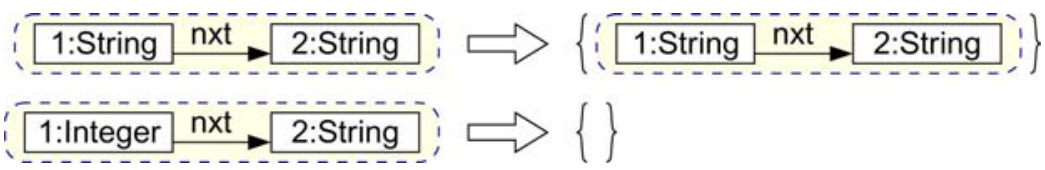

Fig. 8. Flattened edge inheritance constraints

with $G^{n_{1}, e, n_{2}}$ for $n_{1} \in \operatorname{clan}_{I}(\operatorname{src}(e))$ and $n_{2} \in \operatorname{clan}_{I}(\operatorname{tar}(e))$ being the graph consisting of two nodes typed over $n_{1}$ and $n_{2}$, and one edge typed over $e . q_{e, f}$ is the unique type-refining morphism between the source and target graph.

The following theorem states that this formula indeed expresses the satisfaction of the edge inheritance relation, according to Def.13. The proof is omitted here.

Theorem 3 (semantics of edge inheritance). For all type graphs with edge inheritance $T G E I=(T G, I, A)$ and all graphs $G$ clan-typed over $\left(T G,\left.I\right|_{N}, A\right), G$ satisfies TGEI (in the sense of Def. 13) if and only if $G \models{ }^{\mathrm{a}} F_{T G E I}$.

Example 6 (edge inheritance constraints). In Figure 4 (right hand side) we extended the type graph of Fig. 1 with edge type inheritance, depicted by (dashed) inheritance arrows between edges. Hence this type graph expresses (among other things) that an instance may not contain a nxt-edge from a String-typed node to anything but another String-typed node - in particular not to an Integer-typed node — or to a node typed by a subtype of String (of which there are none in this example).

Similarly to the example above, we flatten these graph constraints, i.e., we formulate graph constraints w.r.t. the concrete closure $\widehat{T G I}$ given in Fig. 1 The constraints shown in Fig. 8 are the complete flattening of the first constraint in Fig. 7 Note that the first flattened constraint is always true, and the second describes a handle not allowed by the edge inheritances.

\section{Ensuring Abstract Graph Formulae}

Having defined the concept of abstract graph formulae and shown their utility in formalizing node multiplicities and edge inheritance, we now turn to the issue of ensuring graph constraints (not arbitrary formulae) in a given graph transformation system. A 
graph transformation system is said to ensure a graph constraint if all the graphs that can be derived satisfy the constraint; in other words, if the constraint is an invariant on the derivable graphs. The method for enforcing a constraint is by including appropriate preconditions (which are themselves graph formulae) in the rules, using a technique worked out recently for sub-classes of concrete constraints in [6].

We first define abstract and concrete rules with application conditions, and their matching. The following definition extends that in [2].

Definition 15 (abstract and concrete rules). An abstract rule typed over a type graph $T G I=(T G, I, A)$ with inheritance is given by $p=\left(L \stackrel{l}{\leftarrow} K \stackrel{r}{\rightarrow} R, F_{L}, F_{R}\right)$, where $L, K, R$ are abstract clan-typed graphs, $l$ and $r$ are type-preserving graph morphisms, $F_{L}$ and $F_{R}$ are abstract graph formulae, and $\operatorname{ctp}_{R}^{-1}(A) \subseteq r\left(N_{K}\right)$.

$p$ is called concrete if $L, K, R$ are concrete clan-typed graphs and $F_{L}, F_{R}$ are concrete graph formulae.

Concrete rule $p^{\prime}$ refines abstract rule $p$, if $L^{\prime} \leq L, K^{\prime} \leq K, R^{\prime} \leq R$ and $\left.c t p_{R}^{\prime}\right|_{N_{R}^{\prime}}=$ $\left.\operatorname{ctp}_{R}\right|_{N_{R}^{\prime}}$, and moreover, $F_{L}^{\prime}=$ flat $_{L^{\prime}}\left(F_{L}\right)$ and $F_{R}^{\prime}=$ flat $_{R^{\prime}}\left(F_{R}\right)$. The set of all concrete refinements of an abstract rule $p$ is denoted by $\widehat{p}$.

Example 7 (abstract rules). Fig. 9 shows two abstract rules, modelling the insertion of a new string into an ordered set. InsertFirstStringinserts a string into an empty set (the emptyness is ensured by the application condition), whereas InsertNextString handles the case of a non-empty set: an existing object will become the predecessor of the newly inserted String. Note that, if this existing object already has a successor, application of the rule will violate the multiplicity constraint in Fig. 5. We will show below (Ex. 8) that this condition is obtained automatically by translation from the multiplicity constraints. (Note that Fig. 9 only shows the left and right hand sides; the interface graph can be deduced from the node identities.)

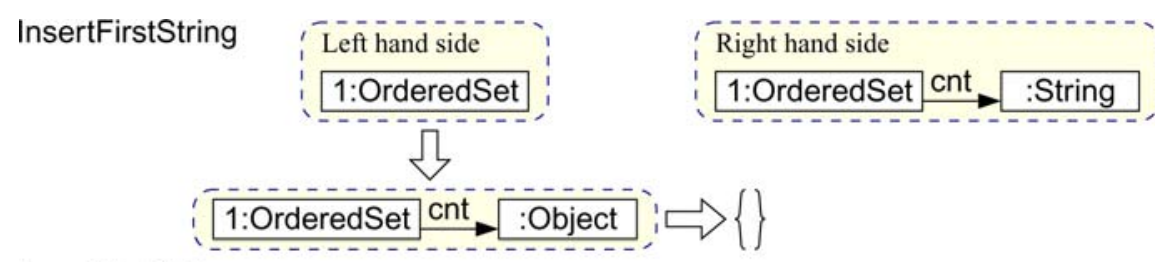

InsertNextString

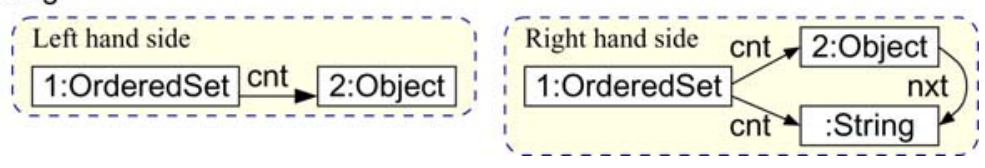

Fig. 9. Abstract rules for inserting a String into an OrderedSet

Definition 16 (rule matching and application). Let $p=\left(L \stackrel{l}{\leftarrow} K \stackrel{r}{\rightarrow} R, F_{L}, F_{R}\right)$ be a derivation rule, $G$ and $H$ concrete clan-typed graphs, and $m: L \rightarrow G$ a type-preserving graph morphism.

- If $p$ is a concrete rule, then $m$ is a match of $p$ in $G$ if 
- $m$ is a match of the untyped rule $\langle L \stackrel{l}{\leftarrow} K \stackrel{r}{\rightarrow} R\rangle$ in the untyped graph $G$, - $m={ }^{\mathrm{c}} F_{L}$.

Given a match $m$, a concrete direct derivation $G \stackrel{p, m}{\Longrightarrow} H$ exists if there is a span of type-preserving morphisms $G \leftarrow D \rightarrow H$ and a co-match $m^{*}: R \rightarrow H$ of $p$ in $H$ that give rise to a derivation in the classical theory of (untyped) graph transformations [5]. The derivation is valid if $m^{*} \models{ }^{\mathrm{c}} F_{R}$.

- If $p$ is an abstract rule, then $m$ is a match of $p$ in $G$ if

- $m$ is a match of the untyped rule $\langle L \stackrel{l}{\leftarrow} K \stackrel{r}{\rightarrow} R\rangle$ in the untyped graph $G$;

- $t_{K}\left(x_{1}\right)=t_{K}\left(x_{2}\right)$ for $t_{K}=\operatorname{ctp}_{G} \circ m \circ l$ and $x_{1}, x_{2} \in N_{K}$ with $r\left(x_{1}\right)=r\left(x_{2}\right)$;

- $m \models{ }^{\mathrm{a}} F_{L}$.

Given a match $m$, an abstract direct derivation $G \stackrel{p, m}{\Longrightarrow} H$ exists if there is a span of type-preserving morphisms $G \leftarrow D \rightarrow H$ and a co-match $m^{*}: R \rightarrow H$ of $p$ in $H$ that give rise to a derivation in the sense of [2]. The derivation is valid if $m^{*} \models{ }^{a} F_{R}$.

The following is the main theorem of [1], extended to the more general application conditions used in the paper. It can be proved using Theorem 1

Theorem 4 (equivalence of abstract and concrete derivations). Given an abstract rule $p_{\mathrm{a}}=\left(L \leftarrow K \rightarrow R, F_{L}, F_{R}\right)$, concrete clantyped graph $G, H$ and a structural match morphism $m: L \rightarrow G$ (i.e. a match with respect to the untyped rule $\langle L \leftarrow K \rightarrow R\rangle$ ), the following statements are equivalent:

1. $m$ is a match of $p_{\mathrm{a}}$ in $G$, yielding a valid abstract direct derivation: $G \stackrel{p_{a}, m}{\Longrightarrow} H$.

2. $m$ is a match of the concrete rule $p_{\mathrm{c}}=\left(L_{\mathrm{c}} \leftarrow K_{\mathrm{c}} \rightarrow R_{\mathrm{c}}, F_{L}^{\mathrm{c}}, F_{R}^{\mathrm{c}}\right)$ in $G$ with $p_{\mathrm{c}} \in \widehat{p_{\mathrm{a}}}$ and $m: L_{\mathrm{c}} \rightarrow_{\mathrm{c}}$ G type-preserving, yielding a valid concrete direct derivation: $G \stackrel{p_{\mathrm{c}}, m}{\Longrightarrow} H$.

In the following, we want to use the translation of graph constraints to application conditions of graph rules as described in [6]. Therefore, we have to restrict the class of graph formulae we use to the ones defined in [6]. If we restrict our concrete graph constraints $G C=(P, C o n)$ to those with $|C o n| \leq 1$, they become equivalent to the positive and negative graph constraints of [6]: the case of $\mid$ Con $\mid=1$ corresponds to positive graph constraints, while the case of $\mid$ Con $\mid=0$ correspond to negative graph constraints 4 Another difference is that, in [6], the morphisms in Con are allowed to be arbitrary, but that does not add expressiveness (although it does add compactness) to those we have defined here, which have injective morphisms only. The following is the relevant result from [6].

Theorem 5 (from concrete constraints to left application conditions). Given a concrete constraint $G C$ and a concrete rule $p=\langle L \leftarrow K \rightarrow R\rangle$, there is a left application condition $\operatorname{acc}_{L}$ such that for all direct derivations $G \stackrel{p, m}{\Longrightarrow} H$ we have: $m \models{ }^{\mathrm{c}} \operatorname{acc}_{L} \Leftrightarrow H \models{ }^{\mathrm{c}} G C$.

By combining this with Theorems 1 and 4 we can prove the following.

\footnotetext{
${ }^{4}$ The result of [6] has since been extended in [8] to and beyond our graph formulae, namely to arbitrarily nested formulae as in [13], which means that the results below also hold for arbitrary formulae.
} 
Concrete application conditions for InsertFirstString

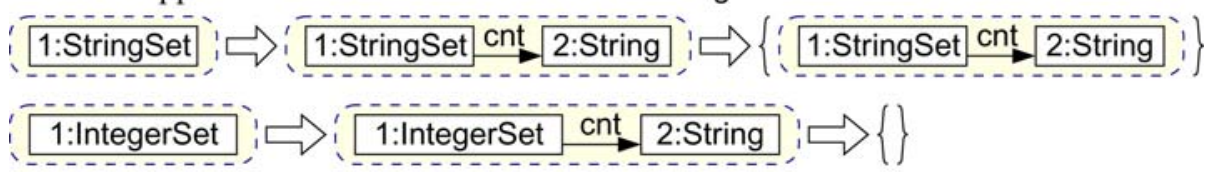

Concrete application condition for InsertNextString

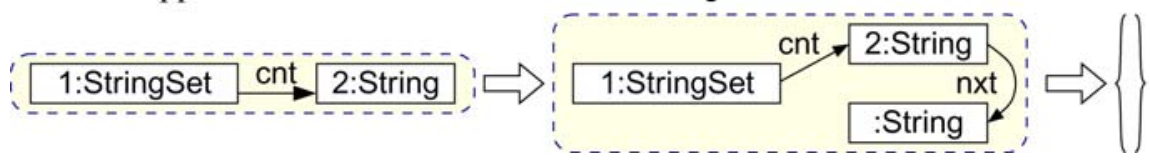

Fig. 10. Derived application conditions

Theorem 6 (from abstract constraints to left application conditions). Given an abstract constraint $G C_{\mathrm{a}}$ and an abstract rule $p_{\mathrm{a}}$ with left hand side $L_{\mathrm{a}}$, there is a set $S$ of concrete application conditions such that for all direct derivations $G \stackrel{p_{a}, m}{\Longrightarrow} H$ we have: $\left(\exists F \in S: m \models^{\mathrm{c}} F\right) \Leftrightarrow H \models^{\mathrm{a}} G C_{\mathrm{a}}$.

Thus, given some abstract graph constraint formula $F_{\text {a }}$ typed over type graph $T G I$ with inheritance, we can flatten it to a concrete graph formula $F_{c}$ as described in Section 3. $F_{\mathrm{c}}$ can be considered as simply typed over concrete closure $\widehat{T G I}$ and translated to a concrete left application condition $\operatorname{acc}_{L}$ that guarantees $F_{\mathrm{a}}$. Note that $\operatorname{acc}_{L}$ is also typed over $\widehat{T G I}$. Unfortunately, there is no straightforward way to translate $\operatorname{acc}_{L}$ to an abstract application condition.

Example 8 (additional application constraints for abstract rules). Consider the constraints in Figs. 5 and 7 respectively and the abstract rules in Fig. 9 Fig. 10 shows some of the elements of $S$ derived for this case according to Th. 6 .

For rule InsertFirstString, the multiplicity constraints (Fig. 5) do not lead to interesting application conditions, since the left-hand side does not contain an Object; but the edge inheritance constraints (Fig.7) induce the two application conditions shown in the figure. These essentially express that the OrderedSet involved has to be a StringSet. For rule InsertNextString, the multiplicity constraint on nxt-edges leads to the third application condition of Fig. 10 (among others). This expresses that the node with identity 2 in the left hand side of the rule (which has the abstract type Object in the rule but concrete type String in the condition) may not have an outgoing nxt-edge; see also Fig. 3.

\section{Conclusions}

In the literature, a variety of formal integrations of object-orientation and formal specification techniques exist. They are considered in the context of precise semantics for UML as well as for precise meta-modeling. It is the declared aim of the precise UML group [12] to come up with a precise standard semantics of the whole language UML, and then to use it for verification purposes. There are various approaches being developed, each 
formalizing certain aspects of UML with the intention of using the resulting precision for formal reasoning. In [3], the authors are especially concerned with the formalization of classes and their relations, inheritance and constraints on the basis of description logics. This work is dedicated entirely to the static part and does not regard the dynamic behavior of objects. Precise meta-modeling is considered in [14], where MOF and graph transformation concepts are integrated. While the aim and the basic ideas are similar to ours, the formalization chosen in [14] is different and not as comprehensive; in particular, it does not deal with constraints.

In addition to formulating a precise semantics, one has also to consider the process by which constraints are enforced. In this paper we have shown one way in which this can be done (by translation to application conditions). We are not aware of other approaches in the literature.

Summarizing, in this paper we have obtained a further, important step of integrating graph transformation with object-orientation concepts: now, type inheritance, constraints, and graph transformation concepts are integrated in one comprehensive formal framework. This offers the possibility to check properties for object-oriented software models. On the meta-model level, the results in our paper can be used to check constraints for model transformation. Further work is needed to carry over other analysis techniques to typed graph transformation with inheritance, to come up with a comprehensive visual and precise framework for object-oriented modeling and meta-modeling.

\section{References}

1. R. Bardohl, H. Ehrig, J. de Lara, O. Runge, G. Taentzer, and I. Weinhold. Node Type Inheritance Concepts for Typed Graph Transformation. Technical Report 2003-19, Technical University Berlin, Dept. of Computer Science, November 2003.

2. R. Bardohl, H. Ehrig, J. de Lara, and G. Taentzer. Integrating Meta Modelling with Graph Transformation for Efficient Visual Language Definition and Model Manipulation. In M. Wermelinger and T. Margaria-Steffens, editors, Fundamental Aspects of Software Engineering (FASE), volume 2984 of $L N C S$. Springer, 2004.

3. A. CalCE, D. Calvanese, G. De Giacomo, and M. Lenzerini. A formal framework for reasoning on UML class diagrams. In Int. Symp. on Methodologies for Intelligent Systems (ISMIS 2002), volume 2366 of $L N C S$, pages 503-513. Springer, 2002.

4. A. Corradini, F. L. Dotti, L. Foss, and L. Ribeiro. Translating Java into graph transformation systems. In Ehrig et al. [7], pages 383-389.

5. A. Corradini, U. Montanari, and F. Rossi. Graph Processes. Special Issue of Fundamenta Informaticae, 26(3,4):241-266, 1996.

6. H. Ehrig, K. Ehrig, A. Habel, and K.-H. Pennemann. Constraints and application conditions: From graphs to high-level structures. In Ehrig et al. [7].

7. H. Ehrig, G. Engels, F. Parisi-Presicce, and G. Rozenberg, editors. Second International Conference on Graph Transformation (ICGT), volume 3256 of LNCS. Springer, 2004.

8. A. Habel. Private communication, 2004.

9. R. Heckel and A. Wagner. Ensuring Consistency of Conditional Graph Grammars - A constructive Approach. Proc. of SEGRAGRA'95 “Graph Rewriting and Computation", Electronic Notes of TCS, 2, 1995. http://www.elsevier.nl/locate/entcs/volume2.html. 
10. T. Mens, S. Demeyer, and D. Janssens. Formalising behaviour preserving program transformations. In A. Corradini, H. Ehrig, H.-J. Kreowski, and G. Rozenberg, editors, First International Conference on Graph Transformation (ICGT), volume 2505 of LNCS, pages 286-301. Springer, 2002.

11. OMG. MDA, MOF, UML and JCL specifications. OMG, 2004. at the OMG web page: http://www.omg.org/.

12. pUML. The precise UML group http://www.puml.org/, 2004.

13. A. Rensink. Representing first-order logic using graphs. In Ehrig et al. [7], pages 319-335.

14. D. Varró and A. Pataricza. VPM: A visual, precise and multilevel metamodeling framework for describing mathematical domains and UML. Journal of Software and Systems Modelling, $1: 1-24,2003$. 